\title{
The Value of Quick Sepsis-Related Organ Failure Assessment Scores in Patients With Acute Pancreatitis Who Present to Emergency Departments: A Three-Year Cohort Study
}

\author{
Alexander Hallac ${ }^{\mathrm{a}, \mathrm{b}}$, Nishant Puria, e, David Applebury ${ }^{\mathrm{a}}$, Kurt Myers ${ }^{\mathrm{b}}$, \\ Parag Dhumal ${ }^{\mathrm{c}}$, Ashish Thatte ${ }^{\mathrm{d}}$, Wichit Srikureja ${ }^{\mathrm{a}}$
}

\begin{abstract}
Background: Distinguishing sepsis from other inflammatory syndromes continues to be a clinical challenge. The goal of risk stratification tools is to differentiate sepsis from other conditions. We compare the ability of quick sepsis-related organ failure assessment (qSOFA) and systemic inflammatory responses syndrome (SIRS) scores to predict prolonged length of stay (LOS) among patients who presented to the emergency department and hospital ward with acute pancreatitis (AP).
\end{abstract}

Methods: We compiled a retrospective database of all adult patients hospitalized for AP during 2015 - 2018 at a single tertiary care center. Independent $t$-tests, Pearson's correlation and multiple regressions were performed with hospital LOS as the dependent variable, versus demographic characteristics and etiology of the pancreatitis as independent variables. Prolonged LOS was defined as $>5$ days.

Results: The sensitivity and specificity of an SIRS score of 2 or greater for the detection of patients with prolonged LOS were $61 \%$ and $80 \%$, respectively. The qSOFA score of 2 or greater corresponded to a diagnosis of significant AP with a specificity of $99 \%$ and a sensitivity of $4 \%$. Multiple regression analysis demonstrated that each point increase in an SIRS score is associated with 2.24 days in additional hospital LOS. Interestingly, SIRS scores were found to correlate with the LOS, but not qSOFA.

Conclusion: The qSOFA is a tool designed to identify patients at high risk of mortality due to sepsis. The data suggest that as with sepsis,

Manuscript submitted February 14, 2019, accepted March 18, 2019

aProvidence Gastroenterology, Spokane, WA, USA

'Internal Medicine Residency, Spokane, WA, USA

${ }^{c}$ College of Business, Economics and Computing, University of Wisconsin, Parkside, WI, USA

${ }^{\mathrm{d} S}$ School of Business Administration, Gonzaga University, Spokane, WA, USA ${ }^{\mathrm{e} C}$ Corresponding Author: Nishant Puri, Providence Gastroenterology, South 105 W. 8th Avenue, Suite 7050, Spokane, WA 99204, USA.

Email: Nishant.Puri@Providence.org

doi: https://doi.org/10.14740/gr1132 patients with AP who are triaged with only qSOFA could be underrecognized and subsequently undertreated. Secondarily, the data suggest that SIRS scoring has the potential to promptly predict how long patients with AP will stay in the hospital.

Keywords: Pancreatitis; Sepsis; Systemic inflammatory response syndrome; Emergency department; Triage

\section{Introduction}

Sepsis is a syndrome of life-threatening organ dysfunction caused by a dysregulated host response to infection. Sepsis is diagnosed in approximately 1.7 million adults in the USA annually and possibly implicated in up to half of all inpatient hospital deaths [1-3]. The high prevalence and mortality of Sepsis syndrome make it the most expensive condition treated in US hospitals with an estimated yearly cost of \$24 billion to the US health care system [4]. The identification of sepsis and characterization of the sepsis syndrome in the current era of highly protocolized patient care is rapidly evolving. Constructing risk stratification tools and care pathways involves integration of translational research, clinical trials and large electronic health record (EHR) datasets. As a result, developing best practices for sepsis is an onerous task with many stakeholders. This task was recently undertaken by a 19-member task force sponsored by the Society of Critical Care Medicine and the European Society of Intensive Care Medicine, and the end result was sepsis-3 [1]. The consensus of the committee included the de-emphasizing of the systemic inflammatory responses syndrome (SIRS) criteria which was established as the prominent precursor/indicator of impending sepsis/infection [5]. Sepsis-3 concluded that utilizing SIRS criteria identifies many patients who are undergoing a physiological response to non-infectious etiologies of sepsis [1,5].

Sepsis-3 proposes the use of a non-laboratory based alternative to the sepsis-related organ failure assessment (SOFA) score named quick SOFA (qSOFA) for mortality prediction in patients thought to be infected. The qSOFAs is to be used immediately upon a patient's presentation to clinicians both 
Table 1. Frequency and Associated Lengths of Hospital Stay for Common Causes of Acute Pancreatitis

\begin{tabular}{lll}
\hline Etiology & Frequency & Mean length of stay (days) \\
\hline Idiopathic $^{\mathrm{a}}$ & 81 & 5.2 \\
Alcohol-induced $^{\mathrm{a}}$ & 75 & 4.8 \\
Choledocholithiasis $^{\mathrm{b}}$ & 58 & 4.5 \\
Mass/obstruction induced $^{\mathrm{b}}$ & 26 & 4.6 \\
Post-ERCP pancreatitis $^{\mathrm{a}}$ & 15 & 4.3 \\
Hypertriglyceridemia $^{\mathrm{a}}$ & 12 & 3.1 \\
Pancreatic divisum $^{\mathrm{b}}$ & 8 & 2.6 \\
Other & 10 & 3.5 \\
\hline Total & 285 & \\
\hline
\end{tabular}

aNon-mechanical. bMechanical. Other: cystic fibrosis, sphincter of Oddi dysfunction, drug-induced and ischemic pancreatitis. ERCP: endoscopic retrograde cholangiopancreatography.

inside and outside of the emergency department (Table 1) [1, 6]. The SOFA and qSOFA are notably meant to be used on patients with a presumed infection; however, on initial examination, infection status is not always clear. Although qSOFA is embraced by much of the professional community, there is concern with the de-emphasis of SIRS most notably by the American College of Chest Physicians (ACCP) [7].

We aim to assess the sensitivity and specificity of initial assessments with qSOFA and SIRS ability to identify and stratify emergency room patients with acute pancreatitis (AP) who had prolonged hospital stay.

\section{Methods}

The Spokane Institutional Review Board approved (which acts in accordance with Helsinki Declaration of 1975 as revised in 2000) the development of a retrospective database comprised of all adult patients (18 years or greater) hospitalized with a diagnosis of AP during 2015 - 2018. The database was created with full ethical compliance as a human study. The Institutional Review Board granted an exception on informed consent given the retrospective nature of the study. The sample size and retrospective time frame was determined based on a predetermined goal of analyzing over 200 subjects. The database was constructed using Current Procedural Terminology (CPT) codes to identify all cases of AP at a single tertiary care center. The diagnosis of AP requires at least two of the following features: characteristic abdominal pain; biochemical evidence of pancreatitis (i.e. amylase or lipase elevated $>3$ times the upper limit of normal); and/or radiographic evidence of pancreatitis on cross-sectional imaging [8,9]. Diagnostic data including vital signs and laboratory studies along with demographic and disposition data were electronically and manually extracted from EHR. Manual data extraction was performed by trained physician researchers who followed strict protocols and did not participate in the admission of patients within the cohort. This retrospective cohort study was reported in accordance with the STROBE statement [10].

The vital signs analyzed were the first recordings obtained in the emergency room and the laboratory data analyzed were the first lab values obtained, frequently within $3 \mathrm{~h}$ of hospital arrival. The patient's Glasgow coma scale and mental status were determined by physician investigators (DA and KM), as well as interpretation of the emergency department documentation, emergency department impressions, hospital history and physical problem lists. In order to prevent potential bias by the physician investigators, a third physician investigator was available for consultation. The qSOFA criteria were defined: altered mental status (AMS); respiratory rate $(\mathrm{RR}) \geq 22 / \mathrm{min}$; and systolic blood pressure (SBP) $\leq 100 \mathrm{~mm} \mathrm{Hg}$. The SIRS criteria were defined: heart rate $(\mathrm{HR}) \geq 90 \mathrm{bpm}$; white blood cell $(\mathrm{WBC}) \geq 12,000 / \mu \mathrm{L}$ or $\leq 4,000 / \mu \mathrm{L} ; \mathrm{RR} \geq 20 / \mathrm{min}$; and temperature $\geq 38.5^{\circ} \mathrm{C}$ or $\leq 36^{\circ} \mathrm{C}$. The aforementioned criteria were used to establish qSOFA and SIRS scores as intended by the authors of each scoring system $[1,5]$. Significant AP was defined as patients who had hospital lengths of stay (LOS) that extended beyond the cohorts median LOS (5 days). The specificity and sensitivity of SIRS and qSOFA's ability to determine which patients would have significant AP were calculated. The etiology of AP was categorized as mechanical (gallstone, mass and ductal disturbance) and non-mechanical (alcohol, post-endoscopic retrograde cholangiopancreatography and hypertriglyceridemia). Cystic fibrosis, sphincter of Oddi dysfunction, drug-induced and ischemic pancreatitis were the least common etiologies and together composed the "other" category.

All statistical analysis was performed using IBM SPSS Statistics for Windows, version 24 (IBM Corp., Armonk, NY, USA). Statistical tests included independent sample $t$-test, Pearson's correlation and multiple regressions performed with LOS as the dependent variable and all other variables as independent variables.

\section{Results}

Three hundred seventy-six patients populated from the EHR search, and 285 had a hospital admission for AP that met the inclusion criteria. The 91 patients who did not meet inclusion criteria lacked sufficient clinical data or documentation while in the emergency department for analysis in this study. The classification of significant AP applied to 23 patients. The 
Table 2. Sensitivity and Specificity of qSOFA in Detecting Prolonged Length of Hospital Stay

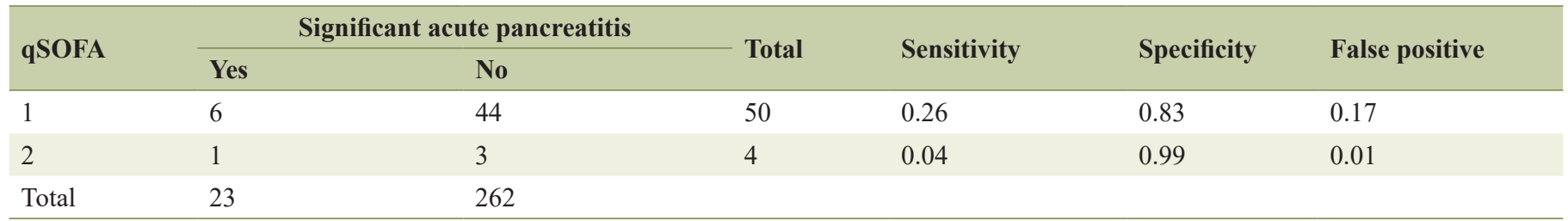

qSOFA: quick sepsis-related organ failure assessment.

mean age of the subjects in the study was 51 years (range: 18 - 98), and a majority were men $(n=147,51.5 \%)$. The mean numbers of days spent by male and female patients are 4.86 and 4.38 , respectively. Independent sample $t$-test showed no difference in the LOS based on gender $(\mathrm{t}=0.45 ; \mathrm{P}=0.64)$. Pearson's correlation between the patients age and LOS was not significant $(\mathrm{r}=0.61 ; \mathrm{P}=0.30)$.

The prevalence of current smokers was $30.5 \%(n=87)$, although $29.4 \%(n=84)$ reported a history of smoking. One-way ANOVA test demonstrated no difference in the average LOS based on current smoking status $(\mathrm{F}=0.27 ; \mathrm{P}=0.76)$.

Seventy-two of the 285 (25.2\%) patients had prior hospital admissions for AP prior to the start of this study. The average numbers of days spent by patients with non-recurrent and recurrent pancreatitis are 4.8 and 3.9, respectively. Independent sample $t$-test showed no difference in the $\operatorname{LOS}(\mathrm{t}=0.78 ; \mathrm{P}=0.43)$.

Alcohol was the most prevalent known etiology of AP ( $\mathrm{n}=$ $75,26 \%)$ followed by gallstone pancreatitis $(n=58,20 \%)$ (Table 1). No specific etiology was found to have a statistically significant impact on the LOS (ANOVA: $\mathrm{F}=0.18 ; \mathrm{P}=0.98$ ) (Table 1). The mean LOS of patients in the non-mechanical and mechanical category are 4.69 and 4.53, respectively. No difference in the LOS was demonstrated between these categories $(\mathrm{t}=0.148$; $\mathrm{P}$ $=0.88$ ). Each patient's entire hospital course was reviewed, and no patients expired secondary to AP during our study.

\section{qSOFA}

No patients in the study met all three qSOFA criteria. The qSOFA score of 2 was present in four patients and corresponded to a diagnosis of significant AP with a specificity of $99 \%$ and a sensitivity of 4\% (Table 2, Fig. 1). No patients with AP secondary to a mechanical etiology met two qSOFA criteria. Twentyone patients with AP induced by a mechanical etiology met one qSOFA criterion, which diagnosed significant AP with a sensitivity of $18 \%$ and a specificity of $81 \%$. The mean qSOFA scores for non-mechanical and mechanical etiologies are 0.93 and 0.83 , and these differences did not influence the LOS $(\mathrm{t}=$ $-0.55 ; \mathrm{P}=0.57)$.

\section{SIRS}

The specificity and sensitivity of an SIRS score of 2 for the detection of patients with significant AP were $61 \%$ and $80 \%$, respectively (Table 3, Fig. 2). The diagnosis of significant AP secondary to a non-mechanical etiology in a patient with an SIRS score of 2 had a sensitivity of $58 \%$ and a specificity of $78 \%$ (Fig. 1). The SIRS score of 2 had a sensitivity of $64 \%$ and a specificity of $83 \%$ in diagnosing significant AP in patients with AP secondary to a mechanical etiology (Fig. 1).

\section{ROC - qSOFA}

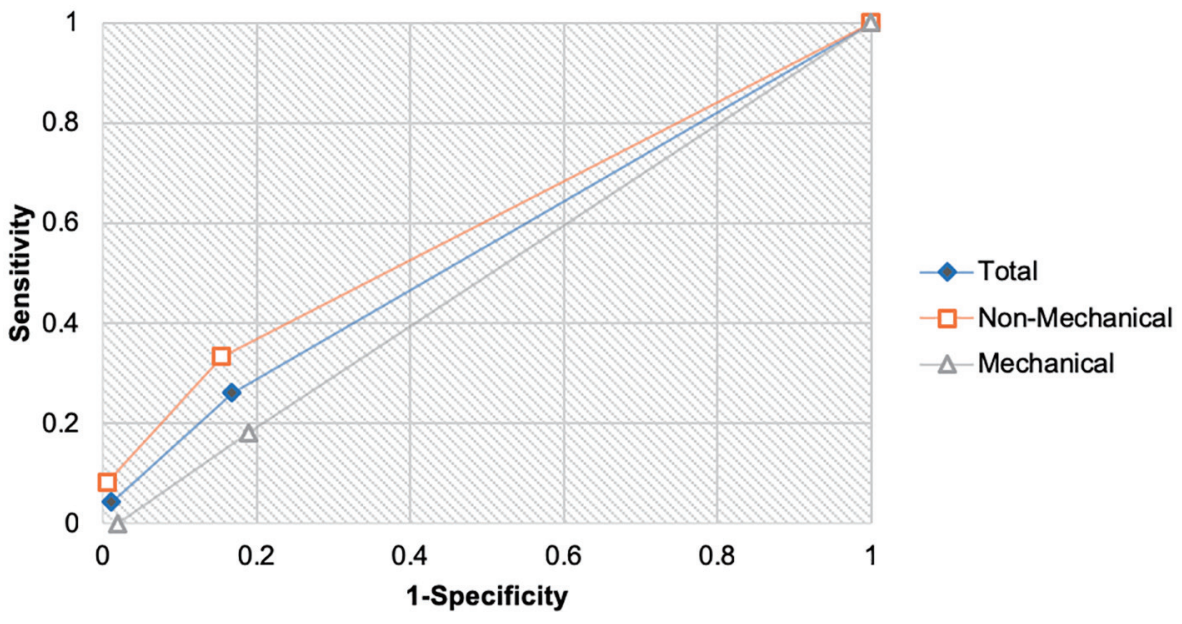

Figure 1. The receiver operating curve demonstrates the sensitivity and specificity of quick sepsis-related organ failure assessment (qSOFA) scoring in detecting significant acute pancreatitis. The underlying mechanism of pancreatitis is mechanical or non-mechanical, and both are individually and collectively analyzed. 
Table 3. Sensitivity and Specificity of the SIRS in Detecting Prolonged Length of Hospital Stay

\begin{tabular}{|c|c|c|c|c|c|c|}
\hline \multirow{2}{*}{ SIRS } & \multicolumn{2}{|c|}{ Significant acute pancreatitis } & \multirow{2}{*}{ Total } & \multirow{2}{*}{ Sensitivity } & \multirow{2}{*}{ Specificity } & \multirow{2}{*}{ False positive } \\
\hline & Yes & No & & & & \\
\hline 1 & 19 & 151 & 170 & 0.83 & 0.42 & 0.58 \\
\hline 3 & 3 & 13 & 16 & 0.13 & 0.95 & 0.05 \\
\hline 4 & 2 & 0 & 2 & 0.09 & 1.00 & 0.00 \\
\hline
\end{tabular}

SIRS: systemic inflammatory responses syndrome.

Multiple regression analysis demonstrated that the effect of qSOFA and SIRS on LOS is significant $\left(\mathrm{R}^{2}=4.8 \% ; \mathrm{F}=\right.$ 8.216; $\mathrm{P}=0.0001)$. Individual testing of the effect that qSOFA had on LOS was not significant $(\mathrm{t}=-0.415 ; \mathrm{P}=0.678)$. Regression testing of SIRS effect on LOS was significant $\left(\mathrm{R}^{2}=5.4 \%\right.$; $\mathrm{F}=16.307 ; \mathrm{P}=0.0001 ; \mathrm{t}=4.038 ; \mathrm{P}=0.0001)$. SIRS scores account for $5.4 \%$ of the variability in the mean LOS of patients with AP. Each point increase in a patient SIRS score adds 2.24 days in hospital LOS.

\section{Discussion}

Although AP is very common and the understanding of the pathophysiology continues to evolve, the management of patients with AP has moved at a glacial speed, remaining largely unchanged for many years.

Over the last half century, numerous risk stratifying tools have been developed, including nine of which Mounzer et al analyzed and determined they had modest diagnostic accuracy (area under curve (AUC) at hospital presentation of $0.6-0.8$ in two cohorts) [11]. Risk stratifying calculations have fallen out of favor in clinical practice; however, categorization of AP is occasionally assigned by the revised Atlanta classification [8, 9]. The development of a superior risk stratifying tool for AP is unlikely until novel diagnostic and therapeutic options for the treatment of AP are enacted.

The absence of a leading AP risk stratification tool and the variability of patient presentation often result in patients being triaged and risk stratified using sepsis scoring systems. Although neither SIRS nor qSOFA is designed to diagnose AP, both sepsis and AP share similar physiology and intravenous fluid resuscitation is a cornerstone of initial management.

Sepsis-3 has many critics including the ACCP who are concerned that the recommendation to retire SIRS criteria could blind clinicians to patients who are early in the continuum of sepsis [7]. An initial qSOFA could falsely reassure clinicians during triage allowing for a delay in performing valuable interventions that may alter the course of a patient's pancreatitis potentially allowing for progression to organ failure.

There is concern that the transition to implementing the qSOFA tool and other elements of the sepsis-3 will negatively affect sepsis management due to the difficulty of appropriately utilizing and implementing this tool in such a complex syndrome. The intended use of qSOFA is to screen for high-risk patients with a presumed infection requiring aggressive man-

\section{ROC - SIRS}

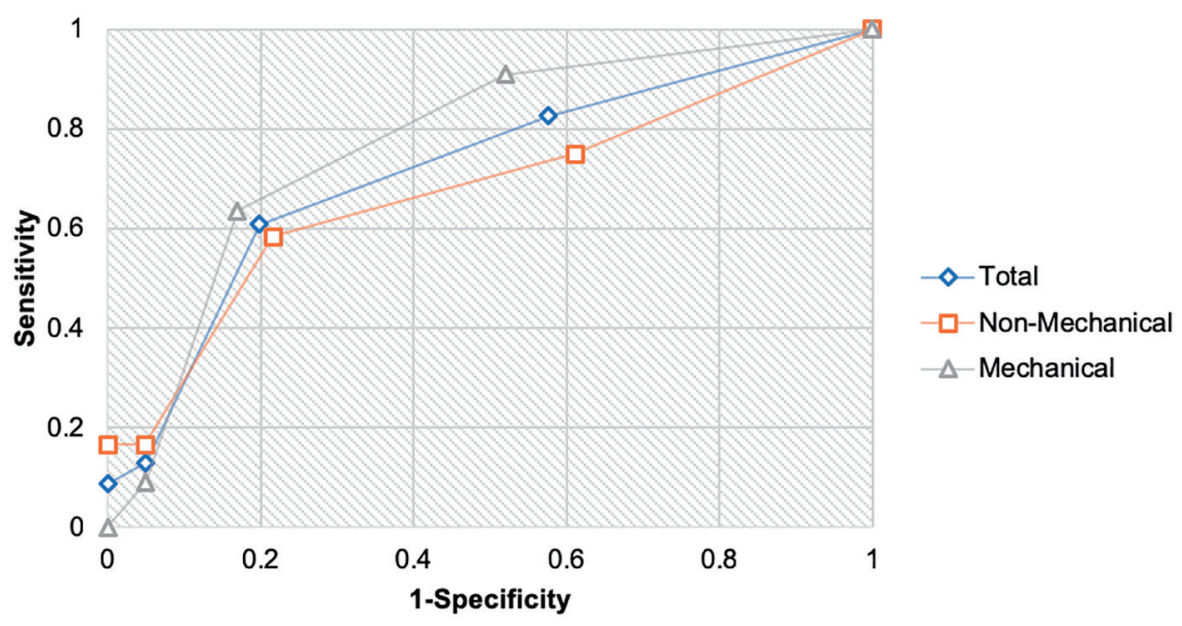

Figure 2. The receiver operating curve demonstrates the sensitivity and specificity of systemic inflammatory response syndrome (SIRS) scoring in detecting significant acute pancreatitis. The underlying mechanism of pancreatitis is mechanical or non-mechanical, and both are individually and collectively analyzed. 
agement in the emergency department and hospital settings; however, it could falsely reassure providers when other pathologies are the causes of illness [1].

Our results demonstrated a sensitivity of $4 \%$ and a specificity of $99 \%$ in the ability of qSOFA to predict patients with significant AP. These same patients in the absence of other diagnostic information would be unlikely to have undergone appropriate intravenous fluid resuscitation due to inadequate identification and triage.

SIRS was superior to qSOFA in predicting patients with prolonged hospital stays as shown in Figure 2. Interestingly, SIRS scores were found to correlate with the LOS in our study, for each point on the SIRS score 2.24 days of hospitalization was predicted. This finding is useful in counseling patients on their predicted hospital course at the time of hospital presentation. The utility of SIRS as a diagnostic tool for significant AP is far from optimal with a sensitivity of $61 \%$ and a specificity $80 \%$.

The limitation of this study beyond the retrospective design is the homogeneity of a single center study. The absence of patients who expired secondary to AP may decrease the generalizability of this study to practitioners at centers with high mortality rates. Although qSOFA is designed for risk stratification of sepsis and is not intended for AP, both pathologies have similar initial management. We believe our data represent the potential risk of undertreating patients with AP who are triaged using the novel qSOFA criteria instead of the SIRS criteria.

\section{Acknowledgments}

None.

\section{Conflict of Interest}

No financial, personal relationships, academic competition or intellectual passion conflict of interest exists for any authors. All authors have reviewed the conflicts of interest statement.

\section{Funding}

None.

\section{Informed Consent}

Not applicable.

\section{Author Contributions}

Conception and design: all authors. Provision of study material or patients: Alexander Hallac, MD; Nishant Puri, MD; David Applebury, DO; Kurt Myers, MD; Wichit Srikureja, MD. Collection and assembly of data: Parag Dhumal; Ashish Thatte; Alexander Hallac, MD; David Applebury, DO; Kurt Myers,
MD. Data analysis and interpretation: Parag Dhumal; Ashish Thatte. Manuscript writing: all authors. Final approval of manuscript: all authors.

\section{References}

1. Singer M, Deutschman CS, Seymour CW, Shankar-Hari M, Annane D, Bauer M, Bellomo R, et al. The Third International Consensus Definitions for Sepsis and Septic Shock (Sepsis-3). JAMA. 2016;315(8):801-810.

2. Rhee C, Dantes R, Epstein L, Murphy DJ, Seymour CW, Iwashyna TJ, Kadri SS, et al. Incidence and Trends of Sepsis in US Hospitals Using Clinical vs Claims Data, 2009-2014. JAMA. 2017;318(13):1241-1249.

3. Liu V, Escobar GJ, Greene JD, Soule J, Whippy A, Angus DC, Iwashyna TJ. Hospital deaths in patients with sepsis from 2 independent cohorts. JAMA. 2014;312(1):90-92.

4. Torio CM, Moore BJ. National inpatient hospital costs. The Most Expensive Conditions by Payer, 2013. HCUP Statistical Brief \#204. Rockville, MD: Agency for Healthcare Research and Quality; May 2016.

5. Bone RC, Balk RA, Cerra FB, Dellinger RP, Fein AM, Knaus WA, Schein RM, et al. Definitions for sepsis and organ failure and guidelines for the use of innovative therapies in sepsis. The ACCP/SCCM Consensus Conference Committee. American College of Chest Physicians/Society of Critical Care Medicine. Chest. 1992;101(6):16441655.

6. Vincent JL, Moreno R, Takala J, Willatts S, De Mendonca A, Bruining $\mathrm{H}$, Reinhart $\mathrm{CK}$, et al. The SOFA (Sepsis-related Organ Failure Assessment) score to describe organ dysfunction/failure. On behalf of the Working Group on Sepsis-Related Problems of the European Society of Intensive Care Medicine. Intensive Care Med. 1996;22(7):707-710.

7. Simpson SQ. New sepsis criteria: a change we should not make. Chest. 2016;149(5):117-118.

8. Banks PA, Bollen TL, Dervenis C, Gooszen HG, Johnson CD, Sarr MG, Tsiotos GG, et al. Classification of acute pancreatitis - 2012: revision of the Atlanta classification and definitions by international consensus. Gut. 2013;62(1):102-111.

9. Crockett SD, Wani S, Gardner TB, Falck-Ytter Y, Barkun AN, American Gastroenterological Association Institute Clinical Guidelines Committee. American Gastroenterological Association Institute Guideline on Initial Management of Acute Pancreatitis. Gastroenterology. 2018;154(4):1096-1101.

10. von Elm E, Altman DG, Egger M, Pocock SJ, Gotzsche PC, Vandenbroucke JP, Initiative S. The Strengthening the Reporting of Observational Studies in Epidemiology (STROBE) statement: guidelines for reporting observational studies. Lancet. 2007;370(9596):1453-1457.

11. Mounzer R, Langmead CJ, Wu BU, Evans AC, Bishehsari F, Muddana V, Singh VK, et al. Comparison of existing clinical scoring systems to predict persistent organ failure in patients with acute pancreatitis. Gastroenterology. 2012;142(7):1476-1482; quiz e1415-1476. 